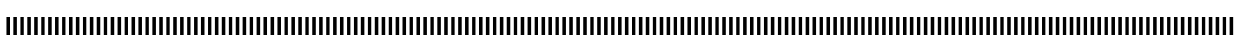

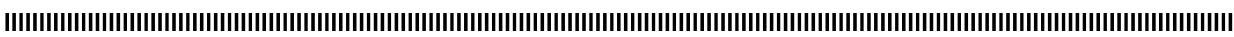

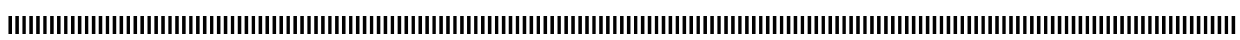

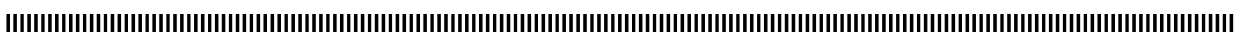

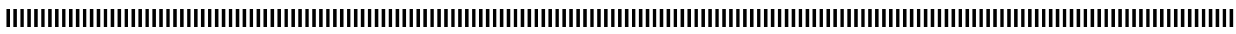

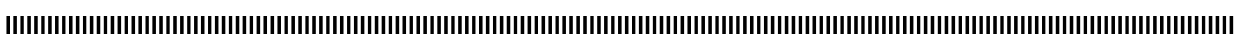

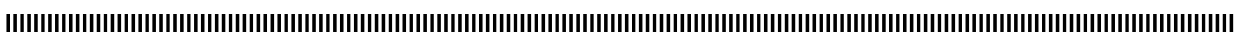

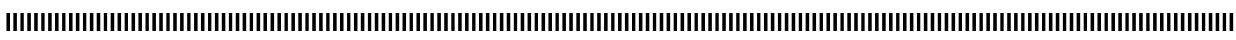

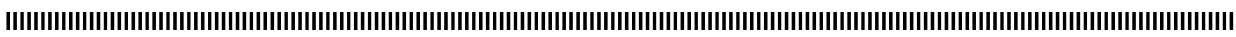

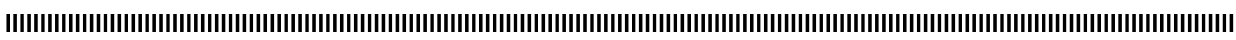

\title{
Calcul des courants de Foucault harmoniques dans des domaines non bornés par un algorithme de point fixe de Cauchy
}

\author{
Faten Jelassi* \\ * LAMSIN, FSB, Jarzouna, 7021, Bizerte Tunisie, faten.jelassi@lamsin.rnu.tn
}

[ ["

RÉSUMÉ. Nous proposons une méthode itérative pour traiter le modèle des courants induits dans un domaine non borné, réécrit au moyen de la technique des éléments finis couplée à une représentation intégrale (désignée en général par la méthode FEM/BEM). La difficulté majeure est la prise en compte numériquement de la condition exacte artificielle et non-locale imposée sur la frontière fictive du domaine tronqué. Un processus itératif est alors mis au point, fondé sur une technique de point fixe de Cauchy, et qui permet une approximation satisfaisante de la solution. Le gain essentiel est qu'à chaque étape nous avons à résoudre un problème posé sur un domaine borné avec une condition locale au bord, ce qui constitue une tâche relativement aisée pour de nombreux codes de calcul conçus pour traiter les problèmes aux limites sur des bornés.

ABSTRACT. We investigate a computing procedure for the unbounded eddy current model put under a coupled finite elements/integral representation form. The exact and non-local artificial condition, enforced on the boundary of the truncated domain, is derived from the simple/double layers potential and the critical point is: how to handle it numerically? An iterating technique, based on the Cauchy fixed point technique, allows us to approximate accurately the solution. The advantage of it is that, at each step, only a bounded eddy current problem with a local condition has to be solved, which is currently carried out by most of the nowadays computing codes conceived to handle value problems on bounded domains.

MOTS-CLÉS : Algorithme de point fixe, électrotechnique, courants de Foucault.

KEYWORDS : Fixed point algorithm, electrotechnique, eddy currents.

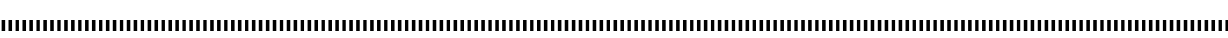

|

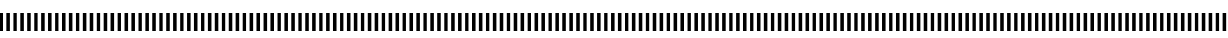

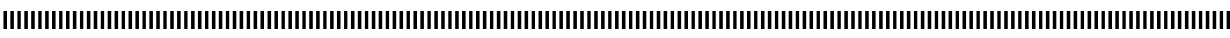

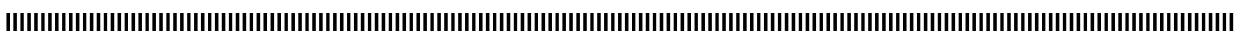
|

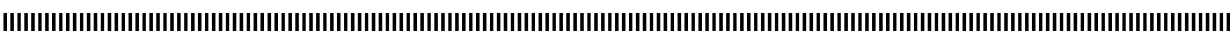

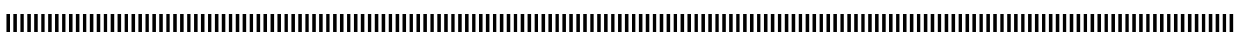
|

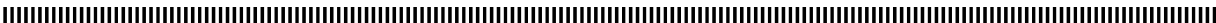




\section{Introduction}

Les équations aux dérivées partielles, posées dans des domaines non bornés, interviennent dans de nombreux secteurs de la physique et de l'industrie. Dans les simulations numériques, la prise en compte du comportement de la solution à l'infini demeure une source de difficulté. Divers outils de calcul ont été mis au point, pendant les dernières décennies, permettant de surmonter cet écueil. Les méthodes les plus populaires, utilisées dans ce genre de discrétisation, consiste à réduire les calculs à des domaines bornés par le biais d'une représentation intégrale adéquate (cf. [11], [8], [4], [7], [14], [9]). Elles sont classées, en général selon la formulation variationnelle qu'elles adoptent. Les premières consistent à écrire une équation intégrale qui est traitée par des éléments frontières (cf. [11]). La matrice de rigidité qui en découle est pleine et mal conditionnée à cause des noyaux singuliers (potentiels de simple couche et de double couche). Les secondes font appel à des conditions artificielles approchées, telles que les conditions de Sommerfeld (cf. [12]). Elles sont discrétisées par des éléments finis usuels, produisant en conséquence des matrices creuses qui, en dépit de leur grande dimension, sont faciles à inverser. Cependant, l'inconvénient réside dans l'imprécision entachant la solution à cause de l'inexactitude de la condition artificielle. Les dernières ont recours à des conditions artificielles exactes définies par une représentation intégrale utilisant des noyaux réguliers (cf. [8]). Elles sont connues sous la dénomination de méthode de couplage élémentsfinis/représentation intégrale (CEFRI) ((FEM/BEM) dans la littérature anglaise). Cette approche bénéficie des avantages des éléments finis usuels et frontières, en évitant leurs faiblesses.

Le problème des courants de Foucault, que nous envisageons d'examiner, est un modèle rencontré couramment en électrotechnique. Des nombreux procédés d'inspection non destructives sont basés sur ce modèle. L'objectif de ce travail consiste à proposer un solveur itératif en vue de résoudre le système issu de l'approximation du modèle des courants induits par une méthode couplée (CEFRI). La condition artificielle exacte écrite sur une frontière de troncature est traitée par un algorithme de type point fixe. Il permet de briser la nature non-locale de cette condition artificielle ce qui conduit à l'inversion d'un problème standard, à chaque itération, peu onéreuse.

Le plan de l'article est le suivant : nous rappelons dans la section 2, les équations du modèle des courants de Foucault dans l'espace. Nous nous plaçons en régime harmonique et dans une géométrie cylindrique rectilinéaire. L'invariance du problème par rapport à la coordonnée longitudinale permet de le réduire en une équation elliptique scalaire posée sur une section transversale du domaine, et dont la seule inconnue est le potentiel magnétique. Nous décrivons, dans la section 3, la méthode de couplage éléments finis/représentation intégrale qui consiste à délimiter le domaine d'étude, tout en tenant compte et de manière efficace de la condition de décroissance à l'infini. Dans la section 4 , nous proposons un algorithme itératif de type point fixe de Cauchy qui s'avère 
compétitif pour la résolution du prototype réduit. Cet algorithme a été analysé en détail pour le problème de Poisson posé sur le complémentaire d'un obstacle dans [1]. Des es-

solution annrochée vers la solution exacte du_nroblème initial Cet article se termine nar une investigation numérique qui conforte les prédictions théoriques.

\section{Modèle réduit des courants de Foucault}

Les conducteurs multi-brins sont souvent utilisés dans la pratique, afin d'augmenter la surface conductrice et atténuer les effets dissipatifs. Nous considérons, ici, un conducteur cylindrique multi-composants; noté $\boldsymbol{\Omega}=\Omega_{\Gamma} \times \mathbb{R}$, sa section transversale $\Omega_{\Gamma}$ est un domaine borné de $\mathbb{R}^{2}$ dont la frontière $\Gamma$ est régulière et divisée en $p^{*}$ composantes connexes notées $\left(\Gamma_{p}\right)_{1 \leq p \leq p^{*}}$ correspondants aux conducteurs $\left(\Omega_{\Gamma_{p}}\right)_{1 \leq p \leq p^{*}}$ (voir Fig. 2, $\Omega_{\Gamma}$ est hachuré).

Les équations aux dérivées partielles régissant le phénomène des courants de Foucault en régime harmonique sont :

$$
\begin{aligned}
& \operatorname{rot} \boldsymbol{H}-\sigma \boldsymbol{E}=\boldsymbol{J}_{\boldsymbol{s}} \quad(\boldsymbol{\Omega}), \\
& \begin{array}{ll}
\operatorname{rot} \boldsymbol{H} & =0 \quad\left(\boldsymbol{\Omega}^{\prime}\right),
\end{array} \\
& i \omega \mu \boldsymbol{H}+\operatorname{rot} \boldsymbol{E}=0 \quad\left(\mathbb{R}^{3}\right), \\
& \operatorname{div}(\mu \boldsymbol{H})=0 \quad\left(\mathbb{R}^{3}\right) .
\end{aligned}
$$

Le domaine $\boldsymbol{\Omega}^{\prime}=\mathbb{R}^{3} \backslash \boldsymbol{\Omega}$ est l'espace libre et sa section transverse est notée $\Omega_{\Gamma}^{\prime}$, $\left(\Omega_{\Gamma} \cup \Omega_{\Gamma}^{\prime}=\mathbb{R}^{2}\right)$. Les champs de vecteurs $\boldsymbol{H}$ et $\boldsymbol{E}$ sont respectivement le champ magnétique et le champ électrique, $\boldsymbol{J}_{s}$ est le vecteur densité du courant source $\left({ }^{1}\right), \omega$ désigne la pulsation et $\sigma\left({ }^{2}\right)$ et $\mu$ constituent respectivement la conductivité électrique et la perméabilité magnétique. Nous supposons, de plus, que $\mu$ est constante dans l'espace libre $\boldsymbol{\Omega}^{\prime}$ et dépend seulement de $\left(x_{1}, x_{2}\right)$ dans $\boldsymbol{\Omega}$ avec $\mu \geq \mu^{*}>0$. Grâce à l'invariance du problème dans la direction longitudinale nous nous proposons de chercher des solutions transverses magnétiques (cf. [3]). Les champs magnétique et électrique sont de la forme

1. Celui-ci est lié au champ électrique par la loi d'Ohm généralisée : $\boldsymbol{J}=\boldsymbol{J}_{\boldsymbol{s}}+\sigma \boldsymbol{E}$, où $\boldsymbol{J}$ est le vecteur densité du courant total.

2. Notons que $\sigma$ est à support compact qui spécifie le conducteur borné $\boldsymbol{\Omega}$. 
$\boldsymbol{H}=\left(H_{x}, H_{y}, 0\right)$ et $\boldsymbol{E}=(0,0, e)$, et le système (1)-(4) est réécrit alors dans sa version bidimensionnelle sous la forme :

$$
\begin{array}{rll}
\sigma e+\operatorname{rot} \boldsymbol{h}=J_{s} & \left(\Omega_{\Gamma}\right), \\
\operatorname{rot} \boldsymbol{h}=0 & \left(\Omega_{\Gamma}^{\prime}\right), \\
i \omega \mu \boldsymbol{h}+\operatorname{rot} e=0 & \left(\Omega_{\Gamma}\right), \\
\operatorname{div}(\mu \boldsymbol{h})=0 & \left(\mathbb{R}^{2}\right),
\end{array}
$$

où $\boldsymbol{h}=\left(H_{x}, H_{y}\right)$ et rot et rot désignent, respectivement, le rotationnel scalaire et vecteur dans $\mathbb{R}^{2}$. La jauge de Coulomb (équation (8)) suggère que $\mu \mathbf{h}$ dérive d'un potentiel $\varphi$ appelé potentiel scalaire magnétique, i.e., $\left(\mu h_{x}, \mu h_{y}\right)=\left(\partial_{x_{2}} \varphi,-\partial_{x_{1}} \varphi\right)$. A l'aide de manipulations algébriques et de géométrie différentielle relativement aisées il est possible d'établir que le potentiel magnétique $\varphi$ satisfait le problème réduit suivant (voir [3], [6]) :

$$
\begin{aligned}
i \omega \sigma \varphi \chi_{\Omega_{\Gamma}}-\operatorname{div}\left(\mu^{-1} \nabla \varphi\right) & =j_{s} \chi_{\Omega_{\Gamma}} & & \left(\mathbb{R}^{2}\right), \\
{[\varphi]=\left[\mu^{-1} \partial_{n} \varphi\right] } & =0 & & (\Gamma), \\
|\varphi(x)| & =\alpha+\mathcal{O}\left(\frac{1}{|x|}\right) & & \text { (à l'infini), }
\end{aligned}
$$

où le symbole $\partial_{n}$ représente la dérivée normale, $\chi_{\Omega_{\Gamma}}$ est la fonction caractéristique de $\Omega_{\Gamma}$. La source $j_{s}$ est une fonction constante par morceaux $\left(j_{s, p}=j_{s \mid \Omega_{\Gamma_{p}}}\right)_{p}$ nulle dans l'espace libre $\Omega_{\Gamma}^{\prime}$. Les $j_{s, p}$ dépendent du courant total $I_{p}$ circulant dans les conducteurs $\Omega_{\Gamma_{p}}$ et sont reliées à $\varphi$ par la relation

$$
j_{s, p}=\frac{1}{\left|\Omega_{\Gamma_{p}}\right|}\left(I_{p}+i \omega\left(1, \sigma_{p} \varphi\right)_{L^{2}\left(\Omega_{\Gamma_{p}}\right)}\right) .
$$

Désormais, le modèle des courants de Foucault est totalement déterminé par l'équation sur le potentiel magnétique :

$$
\begin{array}{rlrl}
i \omega \chi_{\Omega_{\Gamma_{p}}}\left(\sigma_{p} \varphi-\left(1, \sigma_{p} \varphi\right)_{L^{2}\left(\Omega_{\Gamma_{p}}\right)}\right)-\operatorname{div}\left(\mu^{-1} \nabla \varphi\right) & =\chi_{\Omega_{\Gamma_{p}} \frac{I_{p}}{\left|\Omega_{\Gamma_{p}}\right|}} \quad\left(\mathbb{R}^{2}\right), \\
{[\varphi]=\left[\mu^{-1} \partial_{n} \varphi\right]} & =0 & \left(\Gamma_{p}\right), \\
\varphi & =\alpha+\mathcal{O}\left(\frac{1}{|x|}\right) & \text { (à l'infini). }
\end{array}
$$

La sommation d'Einstein est prise en compte ici.

REMARQUE. - En réalité le potentiel $\varphi$ admet un comportement logarithmique à l'infini. Cependant, au moyen de la loi de Biot-Savart, ce terme logarithmique est déterminé de manière explicite. Le potentiel se décompose comme $\varphi=(\log r / 2 \pi) \sum_{p} I_{p}+\mathcal{O}(1)$, à 
l'infini. A l'aide d'un changement d'inconnue nous nous ramenons au système (12)-(14) avec l'hypothèse supplémentaire que $\sum_{p} I_{p}=0 \operatorname{sur} \Omega_{\Gamma_{p}}$.

Pour simplifier la présentation, sans que cela restreigne la généralité de l'étude, nous supposons, dans toute la suite, que le terme source $j_{s}$ est donné par $f$, dont le support est confiné à $\Omega_{\Gamma}$, que la conductivité $\sigma$ est constante dans le conducteur $\Omega_{\Gamma}$ et vaut 1 , de même la pulsation $\omega$ est choisie égale à 1 , et enfin, la permettivité $\mu$ est normalisée de sorte qu'elle soit égale à 1 dans l'espace libre $\Omega_{\Gamma}^{\prime}$, constante par morceaux dans le multi-conducteur $\Omega_{\Gamma}$ et constante sur chaque conducteur $\Omega_{\Gamma_{p}}$. Le potentiel magnétique peut être reformulé ainsi :

$$
\begin{array}{rlc}
i \chi_{\Omega_{\Gamma}} \varphi-\operatorname{div}\left(\mu^{-1} \nabla \varphi\right) & =f \chi_{\Omega_{\Gamma}} \quad\left(\mathbb{R}^{2}\right), \\
\varphi & =\mathcal{O}(1) \quad(\text { à l'infini). }
\end{array}
$$

Le cadre fonctionnel adapté est l'espace de Sobolev à poids, dit aussi de Béppo-Lévi, qui est défini par

$$
W^{1}\left(\mathbb{R}^{2}\right)=\left\{v ; \frac{v}{\sqrt{1+|x|^{2}} \log (2+|x|)} \in L^{2}\left(\mathbb{R}^{2}\right), \quad \nabla v \in L^{2}\left(\mathbb{R}^{2}\right)^{2}\right\},
$$

muni de la norme

$$
\|v\|_{W^{1}}=\left(\|v\|_{L^{2}\left(\Omega_{\Gamma}\right)}^{2}+\|\nabla v\|_{L^{2}\left(\mathbb{R}^{2}\right)}^{2}\right)^{\frac{1}{2}},
$$

qui est une norme équivalente à sa norme naturelle (cf. [11]). L'équivalent variationnel du problème (15)-(16) revient à chercher $\varphi \in W^{1}\left(\mathbb{R}^{2}\right)$ tel que

$$
i \int_{\Omega_{\Gamma}} \varphi \bar{\psi}+\int_{\mathbb{R}^{2}} \mu^{-1} \nabla \varphi \nabla \bar{\psi}=\int_{\Omega_{\Gamma}} f \bar{\psi}, \quad \forall \psi \in V\left(\mathbb{R}^{2}\right),
$$

où $\bar{\psi}$ désigne le conjugué complexe de $\psi$. C'est un problème elliptique et l'existence et l'unicité de (17) sont assurées par le théorème de Lax-Milgram. Notons que la formulation (17) n'est d'aucune utilité pour le calcul scientifique pour des raisons évidentes. Nous avons recours, par conséquent, à la méthode de couplage éléments finis/représentation intégrale (CEFRI).

\section{Méthode couplée (CEFRI).}

La méthode de couplage FEM/BEM permet de tirer profit des avantages des deux techniques CEFRI. Dès lors, la représentation intégrale, fixe le comportement de la solution à l'infini en introduisant une condition à la limite artificielle exacte sur une frontière 
de troncature, rendant le problème couplé accessible au calcul numérique. Cette condition est exprimée sur un bord $\Gamma_{*}$ entourant $\Omega_{\Gamma}$ selon la formule :

$$
\begin{aligned}
\varphi(x) & =\int_{\Gamma_{*}} \varphi\left(\partial_{n_{y}} G\right)(x, y) d \gamma(y)-\int_{\Gamma_{*}}\left(\partial_{n} \varphi\right) G(x, y) d \gamma(y)+c \\
& =M_{\Gamma_{*}}(\varphi)(x)=\left(D_{\Gamma_{*}}(\varphi)-S_{\Gamma_{*}}\left(\partial_{n} \varphi\right)\right)(x)+c, \quad \forall x \notin \Gamma_{*},
\end{aligned}
$$

$G(x, y)=\frac{1}{2 \pi} \ln (|x-y|)$ est la fonction de Green et $\partial_{n_{y}} G(x, y)$ sa dérivée normale par rapport à $y$. Le potentiel mixte $M_{\Gamma_{*}}$ est une combinaison du potentiel de simple couche $S_{\Gamma_{*}}$ et de celui de double couche $D_{\Gamma_{*}}$ et $c$ désigne une constante réelle. La technique de troncature démarre par l'introduction de deux frontières fictives emboîtées $\Gamma_{*}$ et $\Sigma$ entourant l'ensemble des conducteurs $\Omega_{\Gamma}$. En vertu de la relation (18), nous imposons une condition à la limite artificielle de type Neumann sur $\Sigma$ ce qui permet de réécrire le problème réduit dans le domaine borné $\Omega_{\Sigma}$ (domaine intérieur délimité par $\Sigma$ ) sous la forme

$$
\begin{aligned}
i \chi_{\Omega_{\Gamma}} \varphi-\operatorname{div}\left(\mu^{-1} \nabla \varphi\right) & =f \chi_{\Omega_{\Gamma}}\left(\Omega_{\Sigma}\right), \\
\partial_{n} \varphi & =\partial_{n}\left[M_{\Gamma_{*}}(\varphi)\right](\Sigma),
\end{aligned}
$$

La condition artificielle (20) prescrite sur $\Sigma$ est exacte et elle est de type Neumann nonstandard à cause de son caractère non-local provenant du couplage entre $\left(\partial_{n} \varphi\right)_{\mid \Sigma}$ et $\left(\varphi_{\mid \Gamma_{*}},\left(\partial_{n} \varphi\right)_{\mid \Gamma_{*}}\right)$. Soulignons d'emblée, que ce couplage induit des effets indésirables sur la matrice de rigidité du problème couplé obtenu après discrétisation. En plus de son caractère non-hermitien, elle est de structure partiellement creuse avec des blocs pleins liés aux degrés de liberté localisés sur $\Sigma$ et autour de $\Gamma_{*}$. Ceci rend l'inversion du système algébrique assez onéreuse.

REMARQUE. - Le choix d'une condition à la limite de type Dirichlet est possible pour la version tronquée du problème des courants de Foucault. Dans ce cas, la condition artificielle (20) prend la forme suivante

$$
\varphi=M_{\Gamma_{*}}(\varphi), \quad(\Sigma)
$$

\section{Méthode de point fixe de Cauchy}

Afin de briser ce couplage (entre $\Gamma_{*}$ et $\Sigma$ ), nous utilisons un algorithme de point fixe de Cauchy qui permet la construction d'une suite $\left(\varphi^{m}\right)_{m}$ à l'aide d'une récurrence. Le terme $\varphi^{m+1}$ est solution de (19) alors que la condition transparente (20) se transforme comme suit :

$$
\partial_{n} \varphi^{m+1}=\partial_{n}\left[M_{\Gamma_{*}}\left(\varphi^{m}\right)\right]=V_{\Gamma_{*}}\left(\varphi^{m}\right)-K_{\Gamma_{*}}\left(\partial_{n} \varphi^{m}\right) \quad(\Sigma) .
$$


La formulation variationnelle issue du problème (19) et (21) s'appuie sur l'emploi de l'espace de Sobolev $H^{1}\left(\Omega_{\Sigma}\right)$, muni de la norme

$$
\|v\|_{H^{1}}=\left(\|v\|_{L^{2}\left(\Omega_{\Gamma}\right)}^{2}+\|\nabla v\|_{L^{2}\left(\Omega_{\Sigma}\right)}^{2}\right)^{\frac{1}{2}},
$$

qui est une norme équivalente à la norme $\|\cdot\|_{H^{1}\left(\Omega_{\Sigma}\right)}$. Le problème faible consiste à trouver $\varphi^{m+1} \in H\left(\Omega_{\Sigma}\right)$ tel que $: \forall \psi \in H^{1}\left(\Omega_{\Sigma}\right)$

$$
i \int_{\Omega_{\Gamma}} \varphi^{m+1} \bar{\psi}+\int_{\Omega_{\Sigma}} \mu^{-1} \nabla \varphi^{m+1} \nabla \bar{\psi}=\int_{\Omega_{\Gamma}} f \bar{\psi}+\int_{\Sigma} \partial_{n}\left[M_{\Gamma_{*}}\left(\varphi^{m}\right)\right] \bar{\psi} d \gamma(y) .
$$

Le théorème de Lax-Milgram s'applique aussi à ce problème qui admet donc une solution unique dans l'espace $H^{1}\left(\Omega_{\Sigma}\right)$ vérifiant l'estimation suivante :

$$
\left\|\varphi^{m+1}\right\|_{H^{1}\left(\Omega_{\Sigma}\right)} \leq C\left(|f|+\left\|\varphi^{m}\right\|_{H^{1}\left(\Omega_{\Sigma}\right)}\right) .
$$

L'élimination du couplage entre $\Gamma_{*}$ et $\Sigma$ dans le deuxième terme de l'identité (22) s'avère très avantageuse pour l'inversion de la matrice de rigidité de l'équation (22) approchée par la méthode des éléments finis. En effet, cette matrice redevient creuse induisant une économie de stockage et de calcul. Dans la pratique, il est possible d'utiliser des solveurs directs; la factorisation est achevée en pré-processing et, à chaque itération, l'inversion de (22) est effectuée par une méthode de déscente-remontée. Ces avantages sont d'autant plus importants quand le nombre conducteurs augmente. Le choix judicieux pour le processus itératif est de créer des frontières fictives multi-connexes $\Sigma=\cup_{1 \leq p \leq p^{*}} \Sigma_{p}$ et $\Gamma_{*}=\cup_{1 \leq p \leq p^{*}} \Gamma_{*, p}$, les bords $\Gamma_{*, p}$ qui entourent $\Gamma_{p}$ demeurent cantonnés dans $\Omega_{\Sigma_{p}} \backslash \Omega_{\Gamma_{p}}$ (le sous-domaine bordé par $\Sigma_{p}$ et $\Gamma_{p}$ ). L'étude de l'équation (22), dans ce cas de figure, revient à examiner une collection de sous-problèmes variationnels, où chacun est posé dans $\Omega_{\Sigma_{p}}$. Le seul échange nécessaire entre les sous-problèmes concerne la mise à jour de la condition déterminée à chaque itération.

\subsection{Convergence géométrique de la méthode}

Avant de fournir une estimation sur l'erreur $\left(\varphi-\varphi^{m}\right)$, donnons un résultat établissant l'indépendance de l'algorithme du choix de $\Gamma_{*}$, pourvu que celle-ci soit prise dans $\Omega_{\Sigma} \backslash \Omega_{\Gamma}$.

LEMME. - La suite $\left(\varphi^{m}\right)_{m}$ définie par l'algorithme du point fixe de Cauchy (19) et (21) est indépendante du choix de $\Gamma_{*}$.

PrEUVE. - Elle se trouve dans [2]. 
THÉORÈME. - Nous supposons que $\Sigma$ est suffisamment distant de $\Gamma$, alors l'algorithme du point fixe de Cauchy converge avec un taux géométrique, i.e., il existe un réel $\tau \in[0,1[$ tel que

$$
\left\|\varphi-\varphi^{m}\right\|_{H^{1}\left(\Omega_{\Sigma}\right)} \leq C\left(\varphi^{0}\right) \tau^{m}
$$

$C\left(\varphi^{0}\right)$ est une constante strictement positive qui dépend de la donnée initiale.

PREUVE. - Le Lemme (4.1) justifie que nous donnions la preuve seulement dans le cas

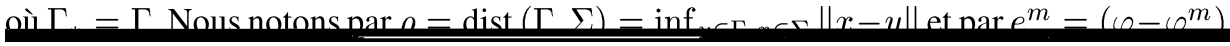
Nous supposons, par ailleurs que $\Gamma$ est de taille 1 , alors que $\Sigma$ est de taille $\rho$ de sorte que la distance qui sépare les deux frontières $\Gamma$ et $\Sigma$ est de l'ordre $\rho$. Alors $e^{m+1}$ est solution du problème variationnel suivant : chercher $e^{m+1} \in H^{1}\left(\Omega_{\Sigma}\right)$ tel que $: \forall \psi \in H^{1}\left(\Omega_{\Sigma}\right)$

$$
\begin{aligned}
i \int_{\Omega_{\Gamma}} e^{m+1} \bar{\psi}+ & \int_{\Omega_{\Sigma}} \mu^{-1} \nabla e^{m+1} \nabla \bar{\psi} \\
& =\int_{\Sigma} K_{\Gamma}\left(e^{m}\right) \bar{\psi} d \gamma(y)-\int_{\Sigma} V_{\Gamma}\left(e^{m}\right) \bar{\psi} d \gamma(y)
\end{aligned}
$$

Par conséquent nous avons

$$
\left\|e^{m+1}\right\|_{H^{1}\left(\Omega_{\Sigma}\right)}^{2} \leq C\left(\left\|K_{\Gamma}\left(e^{m}\right)\right\|_{L^{2}(\Sigma)}+\left\|V_{\Gamma}\left(e^{m}\right)\right\|_{L^{2}(\Sigma)}\right)\left\|e^{m+1}\right\|_{L^{2}(\Sigma)}
$$

Le déroulement de la suite est fortement tributaire de la stablité de l'opérateur trace

$$
\left\|e^{m+1}\right\|_{L^{2}(\Sigma)} \leq C\left\|e^{m+1}\right\|_{H^{1}\left(\Omega_{\Sigma}\right)},
$$

où $C$ est une constante qui ne dépend pas de la taille de $\Sigma$ (la preuve est fournie en annexe dans la section 7). En vertu de l'estimation (26) nous déduisons que

$$
\left\|e^{m+1}\right\|_{H^{1}\left(\Omega_{\Sigma}\right)} \leq C\left(\left\|K_{\Gamma}\left(e^{m}\right)\right\|_{L^{2}(\Sigma)}+\left\|V_{\Gamma}\left(e^{m}\right)\right\|_{L^{2}(\Sigma)}\right)
$$

Avant de borner les termes de droite, observons que les noyaux de Green sont dominés comme suit

$$
\begin{aligned}
\left|\partial_{n_{x}} G(x, y)\right| & \leq \frac{C}{\rho}, \quad \forall x \in \Sigma, \forall y \in \Gamma, \\
\left|\partial_{n_{x}} \partial_{n_{y}} G(x, y)\right| & \leq \frac{C}{\rho^{2}}, \quad \forall x \in \Sigma, \forall y \in \Gamma .
\end{aligned}
$$

Par conséquent, si $\Gamma$ et $\Sigma$ sont distants l'un de l'autre alors il existe un réel $\rho$ suffisamment grand vérifiant : 
Appliquer l'inégalité de Cauchy-Schwarz au potentiel de double et de simple couche conduit à l'estimation

$$
\left|V_{\Gamma}\left(e^{m}\right)(x)\right| \leq \frac{\sqrt{|\Gamma|}}{\rho}\left\|\partial_{n} e^{m}\right\|_{L^{2}(\Gamma)} \quad \text { et } \quad\left|K_{\Gamma}\left(e^{m}\right)\right| \leq \frac{\sqrt{|\Gamma|}}{\rho}\left\|e^{m}\right\|_{L^{2}(\Gamma)} .
$$

En outre, puisque $\Delta e^{m} \in L^{2}\left(\Omega_{\Sigma}\right)$ alors par régularité elliptique (cf. [5]), nous avons pour tout $\mathcal{O}$ un ouvert borné fortement inclus dans $\Omega_{\Sigma}{ }^{3}$, contenant $\Gamma$,

$$
\left\|\partial_{n} e^{m}\right\|_{L^{2}(\Gamma)} \leq C\left\|e^{m}\right\|_{H^{2}(\mathcal{O})} \leq C\left\|e^{m}\right\|_{H^{1}\left(\Omega_{\Sigma}\right)}
$$

Insérant ceci dans (28), il vient que

$$
\left|V_{\Gamma}\left(e^{m}\right)(x)\right| \leq \frac{C}{\rho}\left\|e^{m}\right\|_{H^{1}\left(\Omega_{\Sigma}\right)} \quad \text { et } \quad\left|K_{\Gamma}\left(e^{m}\right)\right| \leq \frac{C}{\rho}\left\|e^{m}\right\|_{H^{1}\left(\Omega_{\Sigma}\right)},
$$

où la constante, ici, est indépendante de $\rho$. La prise en compte de ces majorations dans (27) débouche sur

$$
\left\|e^{m+1}\right\|_{H^{1}\left(\Omega_{\Sigma}\right)} \leq \frac{C}{\sqrt{\rho}}\left\|e^{m}\right\|_{H^{1}\left(\Omega_{\Sigma}\right)} .
$$

Dans le cas où $\Sigma$ est suffisamment loin de $\Gamma$, la constante $\rho$ peut être rendue arbitrairement grande de sorte que $\tau=\frac{C}{\sqrt{\rho}}$ soit dans $[0,1[$. Ce qui complète la preuve.

REMARQUE. - La preuve ne procure aucune information précise sur la distance minimale entre les deux frontières $\Gamma$ et $\Sigma$ garantissant la convergence. Cependant, dans la pratique nous avons observé que cette hypothèse n'est absolument pas nécessaire. $\mathrm{La}$ convergence est vraisemblablement assurée même lorsque la distance entre $\Gamma$ et $\Sigma$ est petite (voir sections 4.2 et 5). Ceci est du à la nature de la condition artificielle. Par contre, dans le cas de la condition de type Dirichlet (3), cette condition sur la topologie de la décomposition du domaine s'avère indispensable pour l'obtention de résultats satisfaisants. En effet, et comme il ressort de l'analyse analytique et des simulations numériques, la version Dirichlet de l'algorithme ne tolère pas un emplacement de la frontière transparente très proche des conducteurs.

\subsection{Examen d'un cas simple avec une symétrie radiale}

Sur une géométrie particulière à symétrie radiale, nous nous proposons d'étudier la fiabilité de l'algorithme de point fixe du problème des courants de Foucault. Une telle étude permet d'examiner de près les contraintes garantissant la convergence, dans le cas où la condition transparente est de type Dirichlet, et de comparer la rapidité de celle-ci

3. Ceci signifie que $\mathcal{O} \subset \overline{\mathcal{O}} \subset \Omega_{\Sigma}$ 
pour les deux versions de l'algorithme (Neumann et Dirichlet) en cas de convergence. Soit un conducteur de section transverse le disque unité, de frontière $\Gamma$ le cercle unité, la frontière intermédiaire $\Gamma_{*}=\Gamma$ et $\Sigma$ est un cercle de rayon $R(1<R)$. Les paramètres physiques $(\mu, \sigma)$ sont tous constants, et nous notons par $\omega \sigma \mu=2 \delta^{2}=2 a^{-2}, \delta$ étant la profondeur de peau et $a$ son inverse. Le conducteur est supposé parcouru par un courant $a^{2} \Re\left(\left(x_{1}+i x_{2}\right)^{k}\right)$ dont la moyenne est nulle. La solution exacte du problème (12)-(14) dans le système de coordonnées polaires $(r, \theta)$ est de la forme $\varphi(r, \theta)=h(r) \cos (k \theta)$ avec

$$
h(r)= \begin{cases}\frac{(i-1) k J_{k}((1-i) a r)}{2 a J_{k-1}((1-i) a)}-r^{k} & r \in(0,1), \\ \left(\frac{(i-1) k J_{k}((1-i) a)}{2 a J_{k-1}((1-i) a)}-\frac{i}{2}\right) r^{-k} & r \in(1, \infty),\end{cases}
$$

où $J_{k}$ représente la fonction de Bessel d'ordre $k$. Sachant que la solution de la version itérative est de la forme $\left(\varphi^{m}\right)_{m}\left(\varphi^{m}=h^{m}(r) \cos (k \theta)\right)$, un calcul simple montre que le taux de convergence de la version Neumann de l'algorithme est

$$
\tau_{N}(k, \delta)=\left|\frac{\gamma(k, \delta)}{R^{2 k}-\gamma(k, \delta)}\right|
$$

$\operatorname{avec} \gamma(k, \delta)=\frac{J_{k+1}((1-i) a)}{J_{k-1}((1-i) a)}$
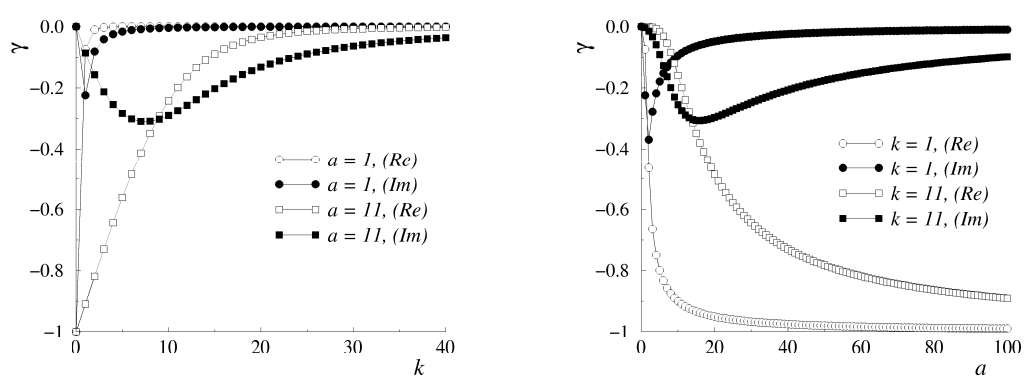

Figure 1. La partie réelle (symboles creux) et la partie imaginaire (symboles pleins) de $\gamma(k, \delta)$ en fonction de $k$ (figure à gauche) et de a (figure à droite), rappelons que le symbole a dans la légende est $\delta^{-1}$

Dès que la partie réelle de $\gamma(k, \delta)$ est négative pour tout choix de $(k, \delta)$, le taux de convergence $\tau_{N}(k, \delta)$ demeure inférieur à 1 en module (voir Fig. 4.2). Il en découle que la suite $\left(\varphi^{m}\right)_{m}$ converge vers la solution exacte $\varphi$ pour toute frontière $\Sigma$, pourvu qu'elle 
soit d'intersection non vide avec $\Gamma$. Une étude comparable pour le cas Dirichlet, aboutit à une expression différente du taux de convergence, sa valeur change en

$$
\tau_{D}(k, \delta)=\left|\frac{\gamma(k, \delta)}{R^{2 k}+\gamma(k, \delta)}\right| .
$$

Nous observons, aussitôt que $\Re(\gamma(k, \delta)) \in]-1,0]$ (voir Fig. 4.2) ce qui nécessite une condition supplémentaire pour assurer la convergence de l'algorithme qui se traduit par $R \geq \sqrt[2 k]{2}$. Dans le cas général, où la densité du courant est à support compact dans le disque unité, la convergence géométrique de l'algorithme est assurée lorsque $R \geq \sqrt{2}$. Nous soulignons, en outre, qu'en cas de convergence, le taux $\tau_{N}(k, \delta)$ est plus petit que $\tau_{D}(k, \delta)$. Ceci illustre l'efficacité de la version Neumann de l'algorithme comparée à celle de Dirichlet.

\section{Discussion numérique}
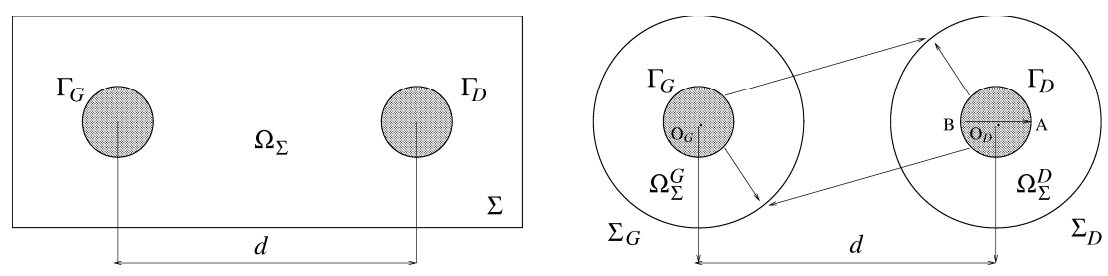

Figure 2. Domaines connexe (à gauche) et non-connexe (à droite). Les flèches indiquent le couplage entre $\Sigma$ et $\Gamma$ induit par la condition artificielle exacte (nous choisissons $\Gamma_{*}=\Gamma$ ).

Nous conduisons une investigation numérique afin de valider la fiabilité du processus itératif proposé à la résolution du modèle réduit des courants de Foucault. Mentionnons, d'ores et déjà, que tous les calculs par éléments finis sont effectués au sein du code MELINA (cf. [10]) après avoir implémenté les procédures nécessaires au calcul des conditions de transparences (le choix retenu, dans ce qui suit est une condition artificielle de type Neumann).

Le premier test consiste à simuler la distribution du courant engendrée par deux conducteurs circulaires portants deux courants constants de charges opposées. Ils présentent une symétrie de position par rapport à l'axe $x_{3}$. Ses sections transverses $\left(x_{1}, x_{2}\right)$, notées $\Omega^{G}$ et $\Omega^{D}$, sont deux disques de rayons $\rho=0.5$ placés au points $O_{G}=(-2,0)$ et $O_{D}=(2,0)$ et séparés par une distance notée $d$ (voir Fig. 2). La donnée $f$ est supportée par les conducteurs, son expression est donnée par

$$
f=\left[\left(x_{1}-2\right) \chi_{\Omega^{D}}-\left(x_{1}+2\right) \chi_{\Omega^{G}}\right]+2 i \delta^{-2}\left[\varphi_{D} \chi_{\Omega^{G}}-\varphi_{G} \chi_{\Omega^{D}}\right],
$$


où $\varphi_{D}$ (resp. $\varphi_{G}$ ) est la solution calculée pour le premier mode $k=1$, à partir de (29) pour $r=x_{1}-2$ (resp. $\left.r=x_{1}+2\right)$. Le symbole $\chi_{\Omega^{D}}$ désigne la fonction caractéristique de $\Omega^{D}$. Le problème possède deux symétries paires par rapport aux deux axes. Dans nos calculs seule la symétrie par rapport à l'axe des $x_{2}$ a été prise en compte. Nous avons mené une simulation de la solution sur deux types de troncature, les unes sont circulaires connexes de centre l'origine et les autres sont circulaires bi-connexes ${ }^{4}$, chacune des composantes est un cercle concentrique avec un conducteur, qui est illustré par la géométrie à droite de Fig. 2. Dans Fig. 3, sont dressées les courbes de convergence pour les troncatures connexes (diagramme de gauche) et non-connexes (diagramme de droite). Au delà de la structure attendue des courbes, au moins lors des premières itérations, qui accrédite une convergence géométrique de l'algorithme nous constatons un meilleur comportement pour les domaines de calcul non-connexes. Pour tenter une explication, nous pensons que pour ces derniers, les frontières artificielles ainsi que la structure des maillages cadrent mieux avec la forme de la solution. En outre, nous n'avons pas à placer $\Sigma$ loin de l'obstacle, étant donné qu'une troncature proche des conducteurs $(R=0.8)$ permet d'obtenir des résultats aussi satisfaisants (avec un coup acceptable d'itérations). D'ailleurs, Tab. 1 illustre bien la bonne qualité de la solution approchée par l'algorithme de point fixe et ce pour la totalité des troncatures.
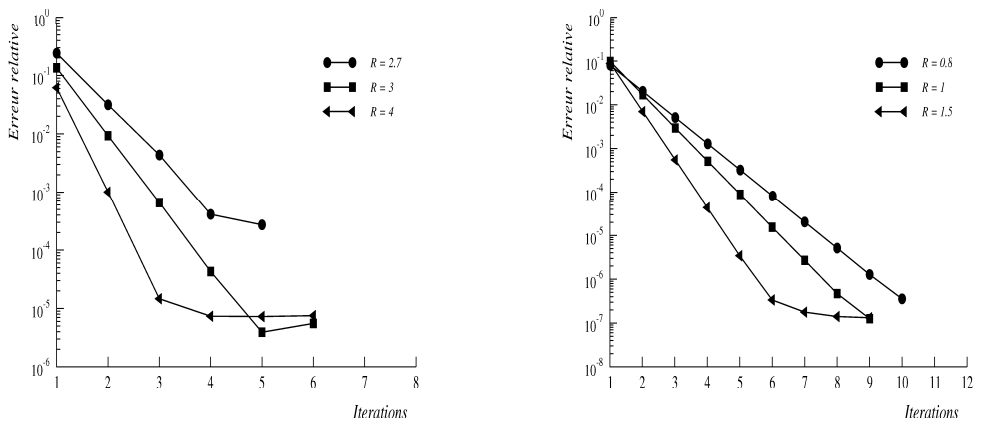

Figure 3. Courbes de convergence de l'algorithme pour le domaine connexe (à gauche) et non-connexe (à droite)(voir Fig. 2).

Le second exemple, pris dans la littérature spécialisée en électrotechnique (cf., e.g., [14]), consiste à simuler la distribution du courant dans l'espace engendré par une paire de conducteurs circulaires véhiculant un courant constant de charges opposées. La dis-

4. II convient de préciser ici que la troncature non-connexe est pertinente du fait de l'absence des modes parasites du problème CEFRI, qui sont les fonctions constantes sur chaque composante connexe du domaine. En effet, le terme source étant à moyenne nulle sur chaque conducteur $\Omega^{G}$ et $\Omega^{D}$, ce qui élimine forcément ces modes parasites. 


\begin{tabular}{|c|c|c|}
\hline$R$ & $\frac{\left\|\varphi^{p}-\varphi\right\|_{\ell^{2}}}{\|\varphi\|_{\ell^{2}}}$ & $\frac{\left\|\varphi^{p}-\varphi\right\|_{\ell \infty}}{\|u\|_{\ell^{\infty}}}$ \\
\hline 2.7 & 0.0089 & 0.013 \\
\hline 3 & 0.0082 & 0.017 \\
\hline 4 & 0.0078 & 0.014 \\
\hline
\end{tabular}

\begin{tabular}{|c|c|c|}
\hline$R$ & $\frac{\left\|\varphi^{p}-u\right\|_{\ell^{2}}}{\|\varphi\|_{\ell^{2}}}$ & $\frac{\left\|\varphi^{p}-\varphi\right\|_{\ell^{\infty}}}{\|u\|_{\ell \infty}}$ \\
\hline 0.8 & 0.0051 & 0.008 \\
\hline 1 & 0.0052 & 0.008 \\
\hline 1.5 & 0.0049 & 0.007 \\
\hline
\end{tabular}

Tableau 1. Ecarts entre $\varphi^{p}$ et $\varphi$ (la solution exacte) par rapport aux normes $\ell^{2}$ et $\ell^{\infty}$ pour des domaines de calcul connexe (à gauche) et non connexe (à droite).

position des conducteurs, supposés infinis, ainsi que la répartition du courant à l'intérieur présentent une invariance par rapport à l'axe $x_{3}$ de sorte que le modèle bidimensionnel est pertinent. Les sections transverses, notées aussi $\Omega^{G}$ et $\Omega^{D}$, sont deux disques de rayons 0.5 , dont les centres sont séparés par une distance $d$ (géométrie à gauche de Fig. 2). L'objectif de l'expérience est, pour une profondeur de peau $\delta=0.1$, de déterminer la structure du vecteur densité du courant $j=\sigma e$, en fonction de la distance $d$. Les calculs sont effectués pour des troncatures rectangulaires connexes à la manière du diagramme à droite de Fig. 2 et en utilisant la symétrie impaire par rapport à l'axe des $x_{2}$ et la symétrie paire par rapport à l'axe des $x_{1}$ (la simulation n'est réalisée, en fait, que pour le quart de la géométrie sélectionnée dans Fig. 2). Pour juger de la fiabilité des résultats obtenus, nous avons représenté dans Fig. 4, la distribution de la densité du courant sur la portion $O_{1} A B O_{1}$ (indiquée dans Fig. 2) en magnitude (diagramme à gauche) et en phase (diagramme à droite), et ce pour les distances $d \in\{1.05,1.5,2.5,5\}$. Le courant est normalisé de sorte qu'il égale l'unité au point $B$. Ces courbes paraissent en conformité avec celles données dans [14]. Ce qui établit, si besoin, la capacité de la méthode itérative à donner des résultats satisfaisants pour des modèles assez pertinents.
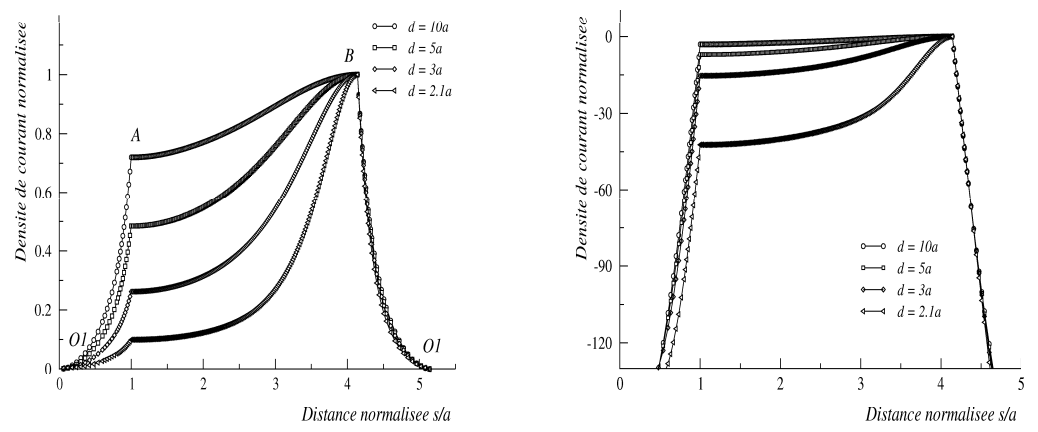

Figure 4. La distribution de la densité de courant en magnitude (à gauche) et en phase (à droite) pour un système de deux conducteurs (Fig. 2) 


\section{Conclusion}

L'objet de cette contribution est de mettre en évidence les performances d'un outil numérique pratique pour traiter le modèle des courants de Foucault. Fondé sur une technique de point fixe de Cauchy, ce processus itératif est avantageux à plusieurs titres : nombre d'itérations relativement bas, gain en temps de calcul... lorsque la condition de transparence, prescrite sur le bord fictif du domaine tronqué, est de type Neumann. Nous insistons tout particulièrement sur un intérêt de cette procédure, qui nous semble majeur. Une simple modification des programmes conçus dans le cas des domaines bornés, permet son adaptation au cas non-borné.

\section{Annexe}

Soit $\Omega$ un ouvert borné de $\mathbb{R}^{2}$, de taille $\varrho$, dont la frontière $\Sigma$ est Lipschitzienne. Notons $\hat{\Omega}=\varrho^{-1} \Omega$, et $\hat{\Sigma}$ sa frontière. D'après [5, Théorème 1.5.1.10], il existe une constante $\hat{C}$ telle que : pour tout $\varepsilon \in] 0,1]$

$$
\|\hat{\varphi}\|_{L^{2}(\hat{\Sigma})} \leq \hat{C}\left(\sqrt[4]{\varepsilon}\|\nabla \hat{\varphi}\|_{L^{2}(\hat{\Omega})}+\frac{1}{\sqrt[4]{\varepsilon}}\|\hat{\varphi}\|_{L^{2}(\hat{\Omega})}\right), \quad \forall \hat{\varphi} \in H^{1}(\hat{\Omega}) .
$$

$\hat{C}$ est indépendante de $\varepsilon$ et de $\rho$. Par un argument de mise à l'échelle, nous obtenons

$$
\|\varphi\|_{L^{2}(\Sigma)} \leq \hat{C}\left(\sqrt[4]{\varepsilon \varrho^{2}}\|\nabla \varphi\|_{L^{2}(\Omega)}+\frac{1}{\sqrt[4]{\varepsilon \varrho^{2}}}\|\varphi\|_{L^{2}(\Omega)}\right), \quad \forall \varphi \in H^{1}(\Omega) .
$$

Choisir $\varepsilon$ de sorte que $\varepsilon \varrho^{2}=1$, conduit à la stabilité

$$
\|\varphi\|_{L^{2}(\Sigma)} \leq C\|\varphi\|_{H^{1}(\Omega)} .
$$

avec $C$ indépendante de la taille de $\Omega$.

\section{Remerciement}

Je tiens à exprimer ma profonde gratitude à mes directeurs de thèse F. Ben Belgacem, du MIP, et N. Gmati, du LAMSIN. Ce travail a bénéficié du soutien du Ministère de la Recherche Scientifique, de la Technologie et du Développement des Compétences (MRSTDC, TUNISIE) dans le cadre du contrat LAB-STI-02. Il a été achevé durant le séjour de F. Ben Belgacem au LAMSIN dans le cadre de l'appel d'offres $2006 \mathrm{du}$ programme "Coopération avec les Chercheurs Tunisiens Résidents à l'Etranger (2006)". 


\section{Bibliographie}

[1] Ben Belgacem, M. Fournié, N. Gmati, F. Jelassi, « Handling boundary conditions at infinity for some exterior problems by the alternating Schwarz method. », C. R. Math. Acad. Sci. Paris, 336, p. 277-282, 2003.

[2] Ben Belgacem, M. Fournié, N. Gmati , F. Jelassi, « On the Schwarz algorithms for the elliptic exterior boundary value problems. ", M2AN Math. Model. Numer. Anal. 39, no. 4, pp. 693-714, 2005.

[3] O. Besson , J. Bourgeois , P.-A. Chevalier, J. Rappaz, R. Touzani, « Numerical modeling of electromagnetic casting processes. ", J. Comp. Phys., V. 92, (1991) 482-507.

[4] M. BonNET, « Equations intégrales et éléments de frontière. », CNRS Eiditions / Eyrolles, Paris, France, 1995.

[5] P. GRISVARD, « Elliptic problems in nonsmooth domains. », Monographs and Studies in Mathematics, 24. Pitman (Advanced Publishing Program), Boston, MA, 1985.

[6] F. JELASSI, « Thèse en préparation ».

[7] R. HIPTMAIR, « Symmetric coupling for eddy current problems. », SIAM. J. NUMER. ANAL., Vol. 40, No. 1, pp. 41-65, 2002.

[8] A. JAMI , M. LENOIR, « A new numerical method for solving exterior linear elliptic problems. ", Lect. Notes in Phys., 90, p. 292-298, Springer, 1979.

[9] J. LIU , J. M. JIN, « A novel hybridization of higher finite element and boundary integral methods for electromagnetic scattering and radiation problems », IEEE transac. ant. propag., V. 49, (2001).

[10] D. MARTIN, « MELINA, Guide de l'utilisateur. », I.R.M.A.R. et E.N.S.T.A. 2000.

[11] J.-C. NÉDÉLEC, « Approximation des équations intégrales en mécanique et en physique. », cours de DEA, CMAP-Ecole Polytechnique, 1977.

[12] A. Sommerfeld, « Lectures on Theoretical Physics. », Academic Press, 1964.

[13] Y. B. YildiR , B. W. KLIMPKe, D. ZhENG. « A computer program for 2D/rs eddy current problem based on boundary element method. », Integrated Engineering Software inc. 46-1313 Border Place, Winnipeg, Manitoba, Canada R3H OX4.

[14] Q. Zheng , J. YI , F. XI , L. MA, « Mutipole theory solution of two-dimensional unbounded eddy current problem. », J. Phys. D : Appl. Phys. 33, p. 195-199, 2000. 